\title{
Experimental characterization of optical-gap solitons in a one-dimensional photonic crystal made of a corrugated semiconductor planar waveguide
}

\author{
S.-P. Gorza, ${ }^{1}$ D. Taillaert, ${ }^{2}$ R. Baets, ${ }^{2}$ B. Maes, ${ }^{2}$ Ph. Emplit, ${ }^{1}$ and M. Haelterman ${ }^{1}$ \\ ${ }^{1}$ Service d'Optique et Acoustique, Université Libre de Bruxelles, 50 Avenue Roosevelt, CP 194/5, B-1050 Bruxelles, Belgium \\ ${ }^{2}$ Department of Information Technology, Ghent University, IMEC, 41, St. Pietersnieuwstraat, B-9000 Gent, Belgium
}

(Received 11 September 2006; published 29 December 2006)

\begin{abstract}
We fully characterize the stationary spatial-gap soliton through the measurement of the phase function of its nearly periodic transverse intensity distribution. The spatial-gap soliton is generated in one-dimensional photonic crystal consisting of a corrugated semiconductor planar optical waveguide. The measured phase function allows us to determine the detuning parameter that provides the position of the gap soliton within the photonic band gap.
\end{abstract}

DOI: 10.1103/PhysRevB.74.235327

PACS number(s): 42.70.Qs, 42.65.Jx, 42.65.Tg, 42.65.Wi

\section{INTRODUCTION}

Nonlinear wave propagation in periodic media has been intensively studied both theoretically and experimentally in the past several years. This interest stems from particular wave propagation properties leading to fascinating phenomena which have no counterpart in homogeneous media. Among these, gap solitons (GS's) have been the focus of considerable attention since their theoretical description two decades ago. ${ }^{1-3}$ These solitons appear as localized waves because the nonlinear self-action suppresses the wave spreading due to the grating action. They have been predicted in various structures such as fiber Bragg gratings and two- and three-dimensional nonlinear photonic crystals ${ }^{4}$ or, outside the field of optics, in Bose-Einstein condenstates in weak periodic potentials. ${ }^{5}$ GS's differ from conventional solitons by the fact that their spectrum lies in the linear forbidden gap of the periodic structure. Moreover, solitons that are slow or even immobile with respect to the grating can be achieved through a proper balance between the two constituting counterpropagating waves. ${ }^{6}$

Optical fibers or channel AlGaAs waveguides with permanently built-in Bragg gratings were the first test beds for the physics of GS's. ${ }^{7-9}$ Yet the impossibility of superimposing the two counterpropagating waves in front of the structure (inherent to the side-on excitation) has made difficult the experimental exploration of the physics of stationary GS's in spite of some suggested solutions. ${ }^{10,11}$

In order to overcome this difficulty, it was proposed in Ref. 12 to expand the one-dimensional (1D) periodic medium by the introduction of an additional homogeneous dimension-say, $z$. In a 2D planar geometry when the refractive index is modulated in one direction - say, $x$ - the diffraction relation of electromagnetic waves [i.e., the relation between the longitudinal $\left(k_{z}\right)$ and transverse $\left(k_{x}\right)$ components of the wave vector] is split up by a forbidden band (photonic band gap). When a Kerr-type nonlinearity is present, the periodic medium supports spatial GS's in the form of a bound state of two beams of different propagation angles. Indeed, the local increase of the refractive index in the center of the beam creates a defect in the linear periodic structure and it is well known that such an inhomogeneity can support spatially localized fields within the band gap. ${ }^{14}$ Actually, the first the- oretical description of spatial GS's has been reported in Ref. 13 in the form of bright staggered solitons. These solitons are realized in arrays of coupled defocusing nonlinear waveguides and can be seen as the counterpart, in nonlinear defocusing media, of the spatial GS's reported in Ref. 12. Spatial GS's are analogous to temporal GS's in 1D periodic media. These spatial solitons can be simply excited by the superposition of two beams (called the forward and backward beams) at the input of a periodic planar waveguide as shown in Fig. 1(a). Therefore, unlike the temporal GS, a stationary GS (with zero transverse velocity with respect to the grating) can be generated. This generation requires two beams of the same power and with opposite incident angles close to the Bragg angles of the structure in such a way that their interference pattern approaches the periodic index profile of the waveguide.

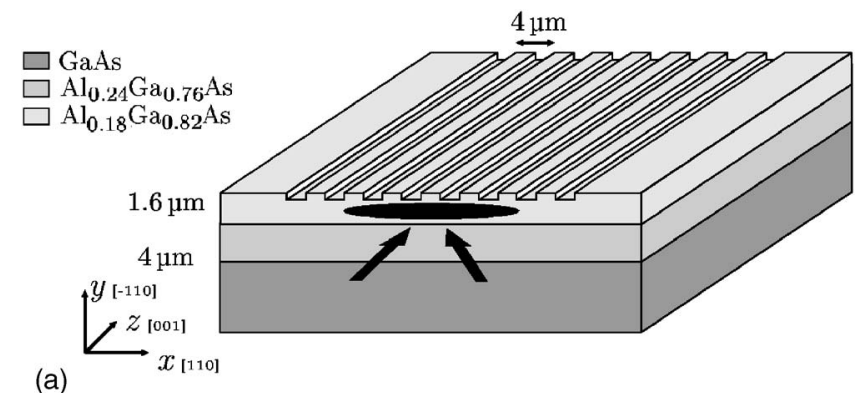

(b)

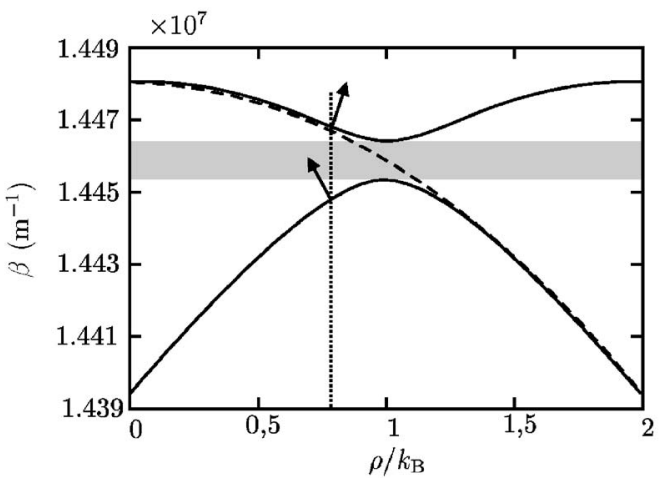

FIG. 1. (a) Planar periodic structure and (b) the associated band diagram. 
Recently light self-confinement effects characteristic of the stationary GS have been observed in photorefractive crystals in defocusing ${ }^{16}$ and focusing ${ }^{17}$ photonic lattices and in semiconductor planar waveguides, ${ }^{18}$ as well as in optical fibers in the temporal domain by means of light-induced temporal gratings. ${ }^{11}$ Note also that in Bose-Einstein condensates, in the limit of the nonlinear Schrödinger equation, bright GS's were experimentally observed as nonspreading wave packets for repulsive atom-atom interactions in a weak periodic potential. ${ }^{5}$ In these previous experiments the GS has been identified only through the observation of the selfconfinement of its constituting wave pair. However, a GS is much more than a simple self-confined state akin to standard spatial solitons ruled by the nonlinear Schrödinger equation. Indeed, the GS constitutes a two-parameter family of solutions in which one of the parameters, called the detuning $\psi$, measures the position of the soliton spectrum with respect to the gap of the linear structure. The detuning $\psi$ also determines the phase function of the nearly periodic transverse intensity distribution of the GS. This phase function-say, $\phi(x)$-represents physically the phase difference between the soliton wave interference pattern and the grating modulation.

The goal of the present work is to show that it is possible to have an access to the detuning parameter $\psi$ of the GS through the measurement of the phase function $\phi(x)$. We provide in this way a crucial method for the full experimental characterization of spatial GS's. The paper is organized as follows: In Sec. II the theoretical model is briefly presented. The linear regime of propagation is discussed in Sec. III. The soliton formation is then considered in Sec. IV, and it is shown how the phase function $\phi(x)$ gives access to the soliton detuning. Our experimental characterization of gap solitons is presented in Sec. V. Eventually, Sec. VI concludes the paper.

\section{THEORETICAL MODEL}

Our experimental demonstration has been performed with laser beams propagating in a periodic planar semiconductor (AlGaAs) waveguide that exhibits a self-focusing Kerr nonlinearity around $1.55 \mu \mathrm{m}$. As shown in Fig. 1(a), the periodicity is obtained by means of a $4-\mu \mathrm{m}$ grating, etched on the top face of the 1.6- $\mu \mathrm{m}$-thick guiding layer. Around the Bragg wave number $k_{\mathrm{B}}=\pi / d$ where $d$ is the grating period, the TE-polarized electromagnetic field

$$
\mathcal{E}(x, z)=\frac{1}{2}\left(E_{+} \mathrm{e}^{i\left(\beta_{0} z+k_{\mathrm{B}} x\right)} 1_{e^{+}}+E_{-} \mathrm{e}^{i\left(\beta_{0} z-k_{\mathrm{B}} x\right)} 1_{e^{-}}\right) \mathrm{e}^{-i \omega t}+\text { c.c. }
$$

is ruled by the nonlinear coupled-mode equations that describe the evolution of a slowly varying envelope of forward $\left(E_{+}\right)$and backward $\left(E_{-}\right)$beams, polarized along the directions $\mathbf{1}_{e^{ \pm}}$included in the $x z$ plane, near the Bragg angle $\theta_{\mathrm{B}}$ $=\operatorname{asin}\left(2 \pi / \lambda k_{\mathrm{B}}\right)$, where $\lambda$ is the wavelength in the vacuum: ${ }^{12,15}$

$$
\frac{\partial E_{+}}{\partial z}+\frac{k_{\mathrm{B}}}{\beta_{0}} \frac{\partial E_{+}}{\partial x}-\frac{i}{2 \beta_{0}} \frac{\partial^{2} E_{+}}{\partial x^{2}}=i \kappa E_{-}+i \gamma\left(\left|E_{+}\right|^{2}+2\left|E_{-}\right|^{2}\right) E_{+},
$$

$$
\frac{\partial E_{-}}{\partial z}-\frac{k_{\mathrm{B}}}{\beta_{0}} \frac{\partial E_{-}}{\partial x}-\frac{i}{2 \beta_{0}} \frac{\partial^{2} E_{-}}{\partial x^{2}}=i \kappa E_{+}+i \gamma\left(\left|E_{-}\right|^{2}+2\left|E_{+}\right|^{2}\right) E_{-},
$$

where $\kappa$ is the coupling constant that describes the effect of the grating on the propagation and $\gamma$ is the waveguide nonlinear Kerr coefficient.

\section{LINEAR PROPAGATION}

In the linear propagation regime (i.e., for $\gamma=0$ ) the coupled-mode equations show that Bragg reflection on the periodic structure opens a photonic band gap that separates the diffraction relation in two bands. This is illustrated in Fig. 1(b) where we plotted in real units the band structure of the grating. The two branches of the diffraction relation correspond to two propagation modes. The propagation directions of these modes are given by the normal to the slope of the corresponding branches [see the arrows in Fig. 1(b)], while the curvature of these branches characterizes the diffraction of light in each mode (i.e., negative diffraction occurs near the upper band edge). ${ }^{20}$ Therefore, when one beam is incident upon the structure, it always excites two modes that travel in opposite directions, as indicated by the black arrows in Fig. 1(b). ${ }^{21}$

This characteristic feature has been observed at the output of our 16-mm-long sample by launching in the waveguide a single TE-polarized laser beam with a width of $80 \mu \mathrm{m}$ at a wavelength of $1.55 \mu \mathrm{m}$. At this wavelength the Bragg angle is $11.2^{\circ}$ in air $\left(3.4^{\circ}\right.$ in the waveguide). The density plot of Fig. 2(a) shows the output beam intensity profile and position versus the input angle $\theta$ (expressed here within the waveguide). We clearly see that for small angles the lateral shift of the beam at the output evolves linearly with the input angle as in a homogeneous medium. Conversely, near the Bragg angle the propagation is strongly affected by the grating: the propagation angle in the periodic medium decreases as the input angle increases and, additionally, the existence of the two bands now manifests itself through the appearance of a second beam corresponding to the lower band of the diffraction relation. At the Bragg angle the two modes travel in the same direction, a feature that allowed us to determine experimentally the Bragg angle with a high accuracy. In Fig. 2(b) we plotted the output beam lateral shift of the upperband Bloch mode as a function of the input angle. Taking the coupling coefficient $\kappa$ as a fit parameter for the comparison with theory, this experimental curve allowed us to get an accurate value of the actual coupling coefficient of the periodic waveguide. We found $\kappa=10300 \mathrm{~m}^{-1}$. Knowledge of this value is essential for the evaluation of the detuning parameter based on the measurement of the phase function $\phi(x)$.

\section{SOLITON PROPAGATION}

When neglecting diffraction, Eqs. (2) admit GS solutions characterized by two parameters $-1<\nu<1$ and $0<\psi<\pi{ }^{6}$ The first one, $\nu$, is related to the soliton transverse velocityi.e., the angle of propagation of the soliton with respect to 

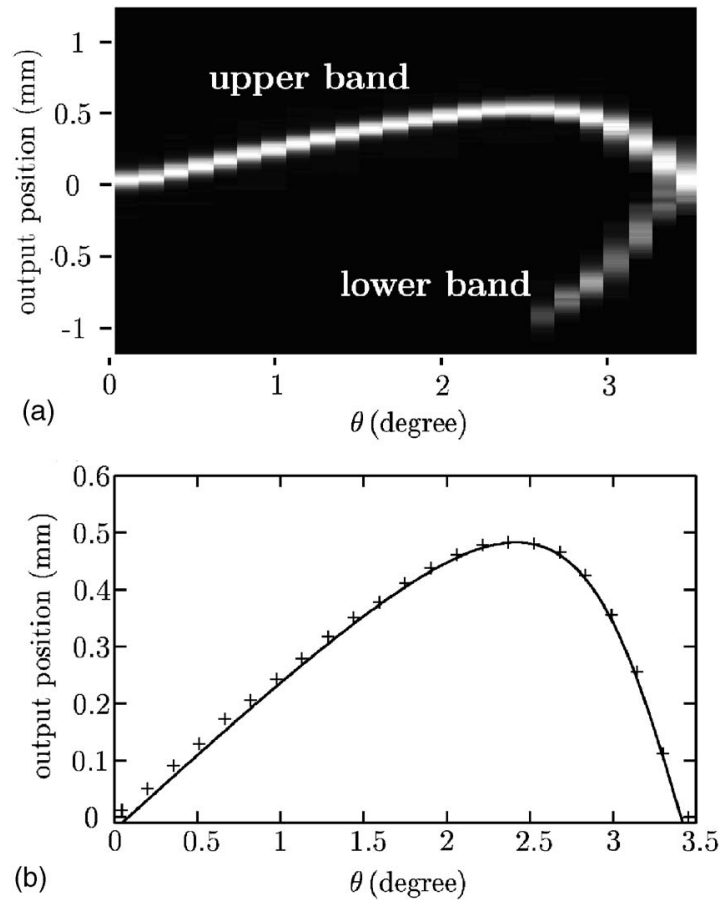

FIG. 2. (a) Intensity profile at the output of a 16-mm-long planar periodic waveguide for one incident beam, as a function of the input angle $\theta$. (b) Lateral shift at the output for the upper-band Bloch mode (crosses). The solid line shows the theoretical shift calculated from the diffraction relation of Eq. (2) with $\kappa=10300 \mathrm{~m}^{-1}$ and $\gamma$ $=0$.

the propagation axis $z$. Here $\nu$ depends on the relative intensity between the forward and backward beams and vanishes when these intensities are equal. In this latter case the soliton travels in the $z$ direction and does not move across the index modulation. This is the analog of the 1D stationary GS of fiber Bragg gratings. The parameter $\psi$ not only determines the soliton width and power, but it also gives the position of the soliton spectrum within the gap [the shift from the center of the band gap is given by $-\kappa \cos (\psi)$ ], which is the reason why it is called the detuning parameter. When $\psi \rightarrow 0$ the soliton is wide, the power is low and the spectrum is near the lower band. Conversely, a narrow soliton with a spectrum close to the upper branch corresponds to a value of $\psi$ close to $\pi$. Additionally, the detuning $\psi$ determines the evolution of the phase $\phi(x)$ of the intensity pattern of the GS. Let us recall that the function $\phi$ must be understood here as being the phase difference between the almost periodic intensity pattern of the soliton and the periodic variations of the waveguide refractive index. As regards stationary GS's, the intensity pattern is out of phase with respect to the index grating in the center of the soliton beam, while in the wings of the beam the intensity pattern and the index grating exhibit a relative phase that progressively tends to $\pi \pm \psi$. More precisely, if the refractive index varies as $\cos \left(2 k_{\mathrm{B}} x\right)$, the oscillating term that modulates the intensity envelope of the GS is $1-\cos \left(2 k_{\mathrm{B}} x+2 \varphi\right)$ where $\varphi(x)=\operatorname{atan}\left[\tanh \left(\kappa \kappa_{k_{\mathrm{B}}} x \sin \psi\right)\right.$ $\left.\times \tan \left(\frac{\psi}{2}\right)\right]$ and the phase function is thus given by $\phi(x)=\pi$ $+2 \varphi(x)$. The solid line in Fig. 3 shows the intensity distribution of a spatial GS with a detuning parameter $\psi=1$. The

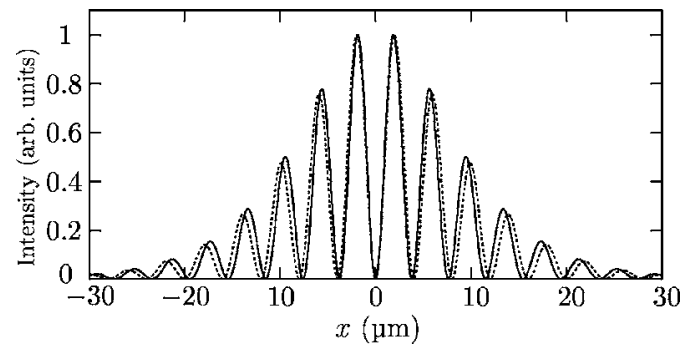

FIG. 3. Intensity profile of a spatial gap soliton (solid line) and a lower-branch Bloch mode modulated by the amplitude envelope of the GS (dotted line). The detuning parameter is $\psi=1$.

intensity distribution of the lower-branch Bloch mode modulated by the amplitude envelope of the GS is also shown for the comparison (dotted line). The phase function $\phi(x)$ manifests itself by the phase difference between these two intensity patterns and constitutes a genuine signature of the nonlinear nature of the GS. As a result, the measurement of the phase function $\phi(x)$ allows, in principle, for the determination of the detuning parameter that in turn provides the position of the soliton spectrum within the band gap. A full experimental characterization of the stationary GS is thus made possible through this phase measurement.

To validate our experimental approach, we numerically investigated the evolution of the phase function $\phi(x)$ along the propagation axis by simulating the model, Eqs. (2), with input Gaussian beams chosen to match at best the GS profile. The phase fronts of the beams are flat so that the initial phase function $\phi(x)$ is always linear. At the soliton power, the numerical simulations show that, over a propagation distance of $4 \mathrm{~mm}$, the slope of the phase function around the beam center tends to and finally reaches the theoretical phase slope of the stationary GS [i.e., $\left.\phi^{\prime}(x=0)=2 \kappa \frac{\beta_{0}}{k_{\mathrm{B}}}(1-\cos \psi)\right]$ independently of the value of the initial phase slope. Conversely, in the beam wings the phase function progressively tends to the expected constant value $\pi \pm \psi$ only over propagation distances much longer than the $4 \mathrm{~mm}$ of our waveguide. This indicates that, in our experimental investigation of the soliton generation, we have to restrict our measures of the phase function to the central part of the beam where the phase is roughly linear. Importantly, these numerical simulations suggest that a GS can be formed by means of two Gaussian beams with flat phase fronts and with limited angular accuracy and propagation length. As a consequence, we can expect to be able to check rigorously the generation of a GS through a full experimental characterization based on the measurement of the function $\phi(x)$.

\section{EXPERIMENT}

In our experiment, we generated a stationary GS by the superposition of two TE-polarized Gaussian beams at the Bragg angle at the input of a 4-mm-long waveguide, as was done in Refs. 17 and 18. The laser source is an optical parametric oscillator pumped by a Ti:sapphire laser producing 130 -fs pulses at $82 \mathrm{MHz}$. We fixed the wavelength at $\lambda$ $=1.57 \mu \mathrm{m}$ so as to obtain a focusing Kerr nonlinearity with 

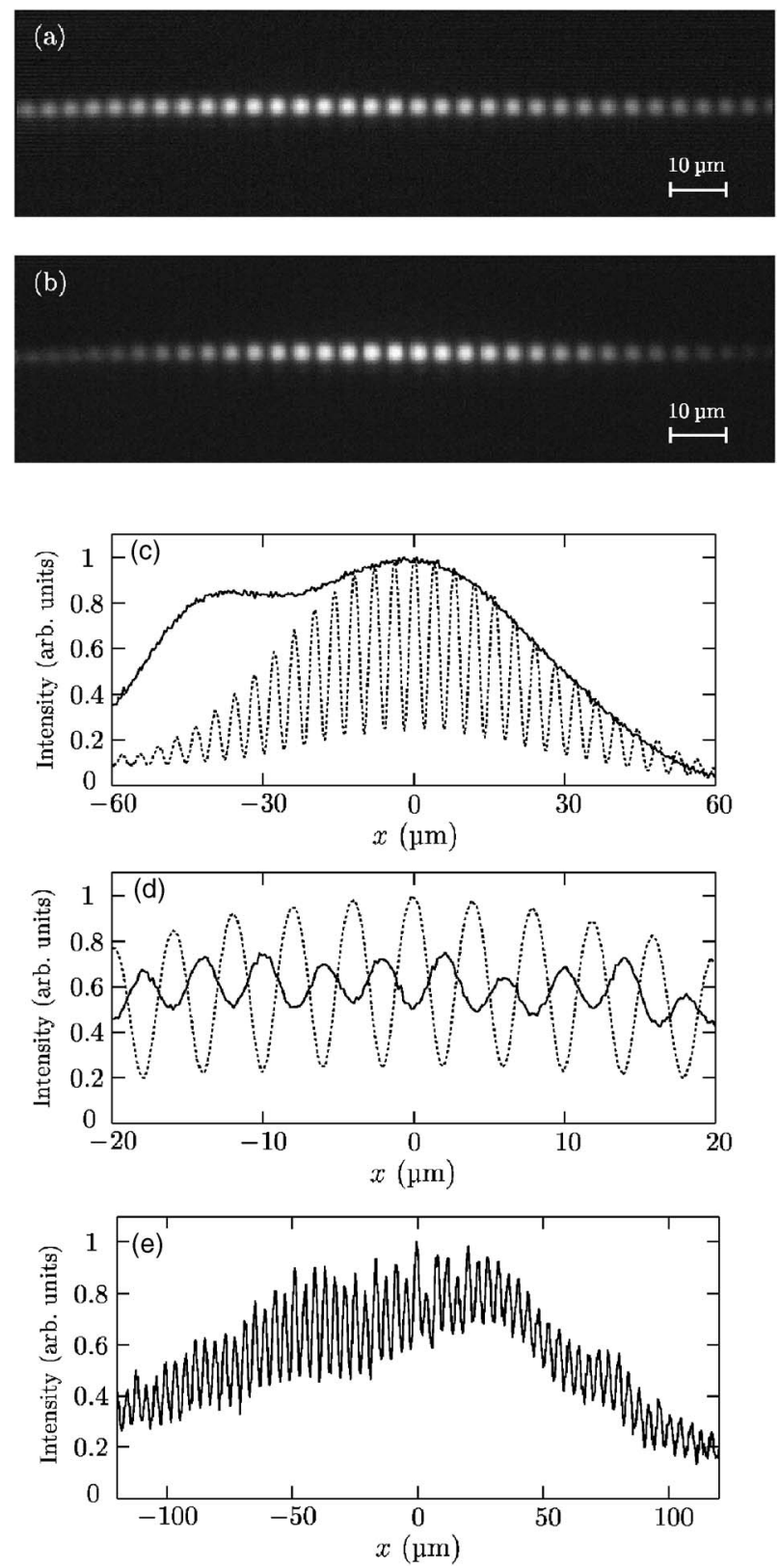

FIG. 4. Output intensity profile at (a) low and (b) high power when a stationary GS is formed (measured output power $P$ $=850 \mathrm{~W}$ ). (c) Intensity profile of one of the two input beams (solid line) and intensity profile at the output of the waveguide at high power when the intensity pattern is out of phase with the index modulation (dotted line). (d) Output intensity profile of the GS beam (dotted line) and the beam used to measure the index modulation (solid line). (e) Output intensity profile at high power when the intensity pattern is in phase with the index modulation.

minimal two-photon absorption since the photon energy is below the energy of the half band gap. ${ }^{19}$ The solid line in Fig. 4(c) shows the intensity profile of one of the two input beams. As can be seen from the asymmetry of the intensity profile, our laser beam is slightly multimodal at the output of the parametric oscillator. However, by injecting this beam into the waveguide outside the grating region, we checked that the asymmetric defect is not coupled to the waveguide

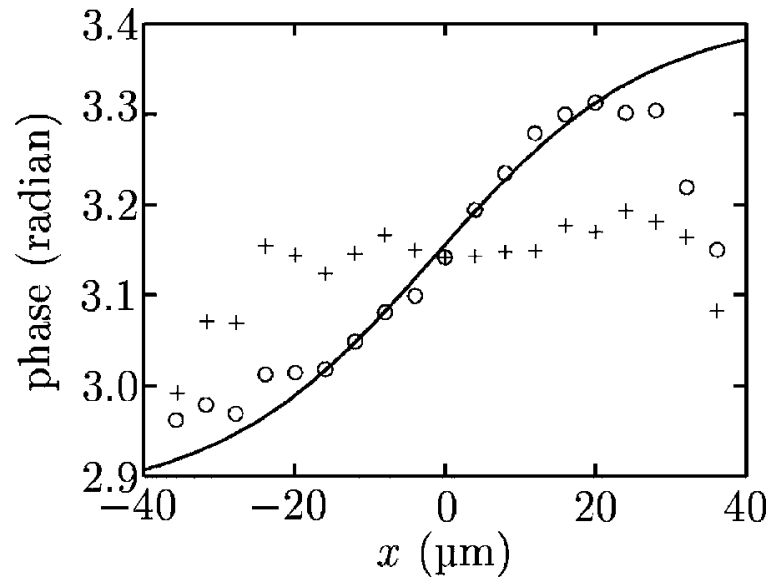

FIG. 5. Output phase function $\phi(x)$ in the conditions of Figs. 4(a) and 4(b), linear regime (crosses) and soliton regime (circles). The solid line shows the theoretical phase function for a stationary GS with a detuning $\psi=0.27$.

mode; i.e., the output beam is always perfectly symmetric, corresponding to a nearly Gaussian input beam of $60 \mu \mathrm{m}$ full width at half maximum (FWHM). The relative phase between the two beams was adjusted to excite a lower branch Bloch mode in the linear regime. In order to visualize the index modulation for the phase slope measurement, we launched in the waveguide a wide beam at normal incidence. At the output, this beam is slightly modulated by the grating and its intensity maxima naturally coincide with the grating index maxima. The location of the index maxima can therefore be recorded to be compared with the soliton intensity maxima. The systematic measurement of the separation distance between the index maxima and the soliton intensity maxima allowed us to reconstruct the full phase function $\phi(x)$ of the stationary GS.

Figure 4(a) shows the intensity profile at the output of the grating, at low power. Due to the effect of the gratinginduced diffraction, the Bloch mode expends up to $95 \mu \mathrm{m}$ FWHM. Conversely, Fig. 4(b) and the dotted line in Fig. 4(c) show that at a measured output peak power of $850 \mathrm{~W}$ the beams keep their initial width of $60 \mu \mathrm{m}$ FWHM. Remembering that the asymmetric defect on the left part of the input intensity profile must be ignored, we observe on the right part of the beam that the intensity profiles of the input and output beams perfectly match, which indicates the formation of the GS. Moreover, we checked that, as predicted by theory, the GS appears when the intensity pattern maxima coincide with the grating index minima, as shown in Fig. 4(d) where the dotted line corresponds to the soliton intensity profile and the solid line corresponds to the intensity profile of the additional beam used to measure the index modulation. When we align the intensity maxima with the index maxima in such a way to excite an upper-branch Bloch mode (at low power), the resulting anomalous diffraction induces a dramatic defocusing at high power. ${ }^{22}$ In this situation we measured, at the soliton power of $850 \mathrm{~W}$, an output beam width of $180 \mu \mathrm{m}$ [see Fig. 4(e)].

Figure 5 shows typical measured phase function $\phi(x)$ in the linear regime and in the soliton regime. In the linear 
regime this phase is almost flat in the center of the beam, as predicted by numerical simulations. This means that the periodicity of the intensity profile is the same as that of the grating, which corresponds to a lower-branch Bloch mode. At the soliton power, the phase function $\phi(x)$ exhibits a linear slope in the center of the beam. The theoretical fit (see Fig. 5) with the phase slope of the GS solution provides, with the parameters of our experiment, the value $\psi=0.27$ for the detuning. This detuning corresponds to a theoretical soliton width of $55 \mu \mathrm{m}$ FWHM, which is in reasonably good agreement with the measured beam width of $60 \mu \mathrm{m}$ FWHM. As regards the soliton power, the detuning of 0.27 corresponds to a theoretical value of $700 \mathrm{~W}$, which has to be compared with the $850 \mathrm{~W}$ used in our experiment. The agreement between theory and experiment is naturally weaker than for the soliton width because of the existence of losses as well as the use of femtosecond pulses instead of the continuous waves considered in theory, which leads to a significant increase of the required power.

\section{CONCLUSIONS}

In conclusion, we have demonstrated the formation of a stationary-gap soliton in a nonlinear periodic planar wave- guide by means of a refined analysis of its transverse intensity pattern. The originality of our approach is that the gap soliton is fully characterized through the measurement of its detuning parameter, which provides its position within the band gap. The agreement between theory and experiment is fully satisfactory in the sense that the measured detuning value is in agreement with the measured soliton width (within reasonable experimental errors). Because we have been able to fully characterize a gap soliton experimentally, our study constitutes a crucial demonstration of the possibility to generate and control gap solitons in 1D nonlinear periodic planar waveguides. Our approach could naturally be applied in other fields of research, such as, for instance, in the experimental study of nonlinear matter waves in weak periodic potentials. ${ }^{5}$

\section{ACKNOWLEDGMENTS}

This work was supported by the Belgian Science Policy Office under Grant No. IAP-V18, the Defay Fund, and the Fonds national de la recherche scientifique (FNRS, Belgium).
${ }^{1}$ W. Chen and D. L. Mills, Phys. Rev. Lett. 58, 160 (1987).

${ }^{2}$ D. N. Christodoulides and R. I. Joseph, Phys. Rev. Lett. 62, 1746 (1989).

${ }^{3}$ A. B. Aceves and S. Wabnitz, Phys. Lett. A 141, 37 (1989).

${ }^{4}$ N. Akozbek and S. John, Phys. Rev. E 57, 2287 (1998).

${ }^{5}$ B. Eiermann, T. Anker, M. Albiez, M. Taglieber, P. Treutlein, K.-P. Marzlin, and M. K. Oberthaler, Phys. Rev. Lett. 92, 230401 (2004).

${ }^{6}$ C. M. de Sterke and J. E. Sipe, in Progress in Optics, edited by E. Wolf (Elsevier, New York, 1994), Vol. XXXIII.

${ }^{7}$ N. G. R. Broderick, in Nonlinear Photonics Crystals, edited by R. E. Slusher and B. J. Eggleton (Springer-Verlag, Berlin, 2003).

${ }^{8}$ B. J. Eggleton, R. E. Slusher, C. M. de Sterke, P. A. Krug, and J. E. Sipe, Phys. Rev. Lett. 76, 1627 (1996).

${ }^{9}$ N. G. R. Broderick, P. Millar, D. J. Richardson, J. S. Aitchison, R. De la Rue, and T. Krauss, Opt. Lett. 25, 740 (2000).

${ }^{10}$ H. G. Winful and V. Perlin, Phys. Rev. Lett. 84, 3586 (2000).

${ }^{11}$ G. Van Simaeys, S. Coen, M. Haelterman, and S. Trillo, Phys. Rev. Lett. 92, 223902 (2004).

${ }^{12}$ J. Feng, Opt. Lett. 18, 1302 (1993).

${ }^{13}$ Y. S. Kivshar, Opt. Lett. 18, 1147 (1993).
${ }^{14}$ A. A. Sukhorukov, and Y. Kivshar, J. Opt. Soc. Am. B 19, 772 (2002).

${ }^{15}$ H. Kogelnik, in Topics in Applied Physics, edited by H. Kogelnik, (Springer-Verlag, Berlin, 1975), Vol. 7; P. Yeh, A. Yariv, and C.-S. Hong, J. Opt. Soc. Am. 67, 423 (1975); D. N. Christodoulides et al., Nature (London) 424, 817 (2003).

${ }^{16}$ J. W. Fleischer, T. Carmon, M. Segev, N. K. Efremidis, and D. N. Christodoulides, Phys. Rev. Lett. 90, 023902 (2003).

${ }^{17}$ D. Neshev, A. A. Sukhorukov, B. Hanna, W. Krolikowski, and Y. S. Kivshar, Phys. Rev. Lett. 93, 083905 (2004).

${ }^{18}$ D. Mandelik, R. Morandotti, J. S. Aitchison, and Y. Silberberg, Phys. Rev. Lett. 92, 093904 (2004).

${ }^{19}$ J. S. Aitchison, D. C. Hutchings, J. U. Kang, G. I. Stegeman, and A. Villeneuve, IEEE J. Quantum Electron. 33, 341 (1997).

${ }^{20}$ H. S. Eisenberg, Y. Silberberg, R. Morandotti, and J. S. Aitchison, Phys. Rev. Lett. 85, 1863 (2000).

${ }^{21}$ D. Mandelik, H. S. Eisenberg, Y. Silberberg, R. Morandotti, and J. S. Aitchison, Phys. Rev. Lett. 90, 053902 (2003).

${ }^{22}$ R. Morandotti, H. S. Eisenberg, Y. Silberberg, M. Sorel, and J. S. Aitchison, Phys. Rev. Lett. 86, 3296 (2001). 